\title{
Linear LMS Compensation for Timing Mismatch in Time-Interleaved ADCs
}

\author{
Damián Marelli, Kaushik Mahata, and Minyue Fu, Fellow, IEEE
}

\begin{abstract}
The time-interleaved architecture permits the implementation of high-frequency analog-to-digital converters (ADCs) by multiplexing the output of several time-shifted low-frequency ADCs. An issue in the design of a time-interleaved ADC is the compensation of timing mismatch, which is the difference between the ideal and real sampling instants. In this paper, we propose a compensation method that, as opposite to existing approaches, does not assume that the input signal is band limited but assumes instead that it has a stationary known power spectrum. The compensation is then designed in a statistically optimal sense. This largely reduces the compensation order required to achieve a given reconstruction accuracy. Also, under the band-limited assumption, the proposed method achieves perfect reconstruction if no constraints are imposed on the order of the compensation. Simulation results show that a rough estimate of the input spectrum can be used without much performance loss, showing that an accurate knowledge of the input spectrum is not necessarily required.
\end{abstract}

Index Terms-Analog-digital conversion (ADC), compensation, least mean squares (LMS) methods, multirate digital filters, sampled-data systems, signal reconstruction, stochastic systems, timing.

\section{INTRODUCTION}

A HIGH-SPEED analog-to-digital converter (ADC) can be realized by using the so-called time-interleaved architecture [1]. In this technique, a $D$-channel time-interleaved ADC consists of $D$ ADCs (called channel ADCs) having the same sampling rate but different sampling phases, as if they were a single converter operating at a sampling rate $D$ times as fast. Recent high-speed ADCs using this technology achieve sampling rates of up to $20 \mathrm{GS} / \mathrm{s}$ [2].

In spite of its conceptual simplicity, a drawback of the time-interleaved technique is that mismatches between different channel ADCs can deteriorate the overall signal-to-noise-and-distortion ratio (SINAD) [3], [4]. Three kinds of mismatches take place in a time-interleaved ADC. Timing mismatch is the difference between the ideal and real sampling instants of each channel ADC, gain mismatch is the difference between their gains, from analog input to

Manuscript received August 17, 2008; revised December 02, 2008. First published February 20, 2009; current version published November 04, 2009. This work was supported by EU Marie Curie Fellowship MEIF-CT-2006-023728. This paper was recommended by Assoicate Editor G. Manganaro.

D. Marelli was with the Numerical Harmonic Analysis Group, Faculty of Mathematics, University of Vienna, Vienna, Austria. He is now with the School of Electrical Engineering and Computer Science, University of Newcastle, Callaghan, NSW 2308, Australia (e-mail: Damian.Marelli@newcastle.edu.au).

K. Mahata and M. Fu are with the School of Electrical Engineering and Computer Science, University of Newcastle, Callaghan, NSW 2308, Australia (e-mail: Kaushik.Mahata@newcastle.edu.au; Minyue.Fu@newcastle.edu.au).

Digital Object Identifier 10.1109/TCSI.2009.2015730 digital output, and offset mismatch is the difference between their ground values. In this paper, we consider the problem of timing-mismatch compensation.

A first step in a timing-mismatch compensation strategy involves estimating the mismatches. The two main approaches for doing so are called foreground and background techniques. In foreground techniques, the sampling times are estimated using a test signal, which can be a ramp [5] or a sinusoid [6], [7]. In order to avoid interrupting the normal ADC operation, the test signal can be mixed with the input signal and then separated from the obtained samples [5]. However, this approach reduces the available dynamic range of the ADC. This is avoided in background techniques, where the sample times are estimated from the digital outputs of the channel ADCs, while in normal operation. Different approaches for doing so are available. In [8] and [9], the sample intervals between every two consecutive channel ADCs are estimated using the mean-square value of the difference between their samples. A different approach uses oversampling to guarantee that the input signal has no spectral components on a frequency band around the Nyquist frequency. The sampling times can then be estimated by requiring that the reconstructed samples satisfy this spectral property [10], [11]. Finally, a background-estimation technique for digital data signals is proposed in [12], where the estimation is done by minimizing the difference between the observed samples and their values obtained after a slicer (i.e., after detecting their digital values).

Once the timing mismatches have been estimated, this information is used to design a compensator. There are two main options for doing so. In the first option, the sampling clock of each ADC is adjusted to eliminate the timing mismatch [13]. A drawback of this approach is that it requires some means of controlling the sampling clock. This not only complicates the hardware implementation but also tends to increase the random sampling jitter. To avoid these problems, the second option corrects the timing mismatches by digitally processing the channel ADC outputs to interpolate the sample values that would have occurred at the ideal sample times. This can be done using different available approaches. An early method carries out the compensation in the frequency domain [14]. The drawback of this approach is that, being a frequency-domain method, it requires the batch processing of the "whole history" of the sampled signal, which prevents its usage for real-time applications. To get around this issue, a number of methods have been proposed, which carry out the compensation using multirate filter banks [15]-[17]. We describe these methods in some detail in Section III.

Obviously, a timing-mismatch compensator needs to be redesigned when the sampling pattern changes due to, e.g., 
component aging and temperature variations. A desirable property for a compensation technique is that this redesign can be efficiently done so that it can be easily done online. Generally speaking, this is addressed by expressing the compensation filters, associated to all possible timing mismatches, as linear combinations of a small number of fixed filters, weighted by coefficients that depend on the mismatches. In [16] and [18], the fixed filters are optimally designed in a least squares or min-max sense. Since this technique leads to a high design complexity when the number of channels is large, a suboptimal solution was proposed in [19] and [20] using the Taylor expansion of the compensation filters with respect to the channel timing mismatches.

A common assumption of the timing-mismatch compensation methods in [15]-[17] is that the signal to be sampled is band limited. Under this assumption, all aforementioned methods are able to achieve perfect reconstruction (i.e., the contribution of the timing mismatch to the overall SINAD is completely removed) if the order of the compensation can be arbitrarily large. However, the band-limited assumption may not only be unrealistic in many applications but also, as illustrated in Section VI-D, results in compensation filters with a very slow impulse response decay. Consequently, accuracy can only be achieved with compensations having very high orders. This is particularly important in real-time applications since, due to the high sampling rate, a compensation of very high order can be computationally unaffordable. To address this issue, in this paper, we drop the band-limited assumption, and we design the compensation in a statistically optimal [least mean squares (LMS)] sense, using some knowledge (see hereinafter) on the power spectrum of the input signal. This leads to compensation filters having a much faster decay rate, largely reducing the compensation order for a given accuracy. Furthermore, the design can be optimally done by taking into account a constraint on the compensation order. The proposed compensation is derived as a (matrix) Wiener filter [21], [22]. However, we point out in Section IV-C that, like the methods in [15]-[17], it is equivalent to a filter-bank compensation. Since the proposed method is designed for non-band-limited signals, it obviously cannot achieve perfect reconstruction in general. However, again, like the methods in [15]-[17], it does so in the band-limited case, provided that the order of the compensator is not restricted.

To carry out the proposed LMS design, we need to do some assumption on the power spectrum of the input signal. In this sense, there are three different scenarios in the following.

1) In some applications, this information is known a priori by the user, e.g., digital communication applications, where the signal is modulated data with a known stationary spectrum.

2) If the input power spectrum is time variant or unknown to the user, it can be estimated online using a standard technique. These techniques are divided into indirect and direct ones [23]. Indirect algorithms estimate a discrete-time spectrum, which is then converted into continuous time [24], while direct algorithms "directly" estimate the parameters of a continuous-time spectrum. A direct algorithm for continuous-time autoregressive models can be

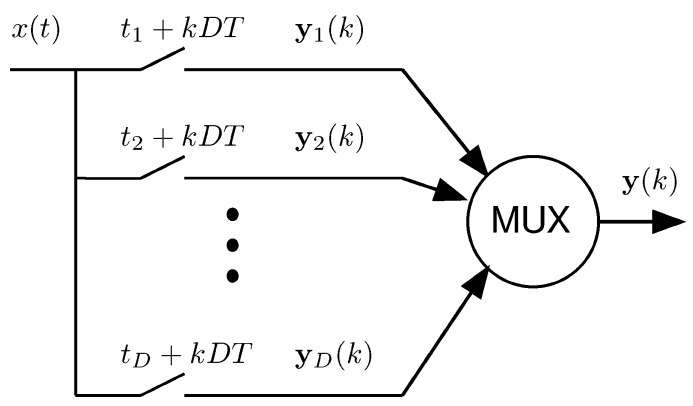

Fig. 1. Time-interleaved ADC scheme.

obtained by approximating the differentiation operation in discrete time [25]. However, the extension of this technique to continuous-time autoregressive moving average models is nontrivial. In [26], this is done by fitting the parameters of the continuous-time spectrum to match its estimate at multiples of the sampling period obtained from the available samples. To reduce the amount of samples required to achieve an accurate spectrum estimate, at the expense of extra hardware, the method in [27] uses a continuous-time multiple-output filter (called input-to-state filter) to generate a continuous-time vector signal whose covariance matrix is used for estimation.

3) If the input spectrum is not known and an estimation algorithm cannot be afforded, a nominal spectrum can be used. Simulation results show that, while the proposed method loses some performance when a rough estimate of the input power spectrum is used, it still largely outperforms the available methods. This shows that an accurate knowledge of the input spectrum is not necessary.

The rest of this paper is organized as follows. We give an overview of time-interleaved ADCs in Section II and of the methods for timing-mismatch compensation in Section III. In Section IV, we introduce the proposed method, and we show how it is equivalent to a filter-bank compensation. We consider two cases: The first one does not impose any constraint on the order of the compensator, while the second one does. In Section V, we state some properties of the proposed method when the input power spectrum is assumed to be band limited. Finally, some simulation results are presented in Section VI, and concluding comments are given in Section VII.

\section{TIME-INTERLEAVED ADCs}

The time-interleaved ADC scheme is shown in Fig. 1. The continuous-time input signal $x(t)$ is sampled using $D$ slowrate channel $\mathrm{ADCs}$, operating at sampling frequency $1 / D T$, but having different sampling phases. The $d$ th ADC's sampling phase is denoted by $t_{d}$, i.e., its output $\mathbf{y}_{d}(k)$ is given by

$$
\mathbf{y}_{d}(k)=x\left(t_{d}+k D T\right)
$$

The outputs $\mathbf{y}_{d}(k), d=1, \ldots, D$, are then multiplexed to generate the time-interleaved ADC output $\mathbf{y}(k)$, which has an average rate of $1 / T$.

Obviously, if the sampling phases $t_{d}$ 's satisfy

$$
t_{d}=(d-1) T, \quad d=1, \ldots, D
$$




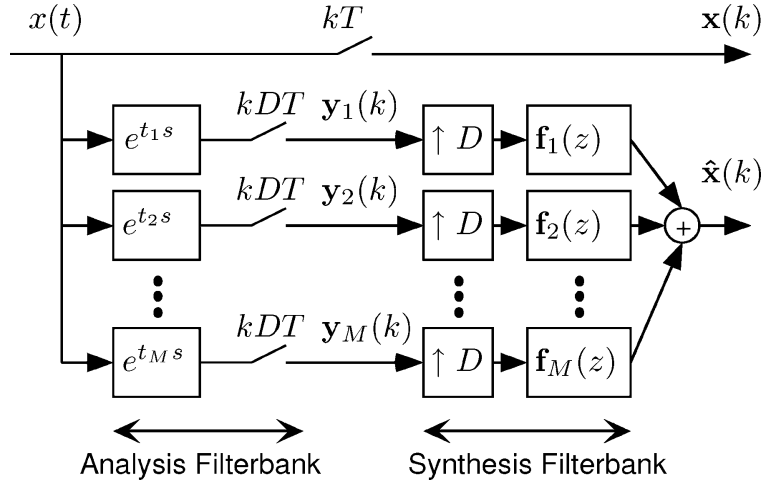

Fig. 2. Filter-bank-based timing-mismatch compensation scheme.

then $\mathbf{y}(k)$ equals the samples $\mathbf{x}(k)$ that would be obtained by using a fast $\mathrm{ADC}$ of sampling frequency $1 / T$, i.e.,

$$
\mathbf{y}(k)=\mathbf{x}(k):=x(k T)
$$

However, (2) cannot be guaranteed always in practice, and therefore, an estimate $\hat{\mathbf{x}}(k)$ of the regular samples $\mathbf{x}(k)$ needs to be constructed from the available samples $\mathbf{y}_{d}(k), d=1, \ldots, D$.

\section{MethodS FOR TIMING-Mismatch COMPENSATION}

As mentioned in Section I, a number of filter-bank-based methods have been proposed to address the timing-mismatch compensation problem described in Section II. A general scheme describing all of them is shown in Fig. 2. In this scheme, the arrangement of $M$-channel ADCs, with its associated sampling phases, is considered as an analysis filter bank with continuous-time input and discrete-time output, formed by the filters $e^{t_{m} s}, m=1, \ldots, M$ (with $s$ denoting the Laplace variable), whose outputs are synchronously sampled at frequency $1 / D T$. The compensation is then done by using a synthesis filter bank, which is implemented by an upsampling operation (i.e., $D-1$ zero-valued samples are added between every two samples), then filtering each component using the array of filters $\mathbf{f}_{m}(z), m=1, \ldots, M$, and finally adding together all the resulting signals.

The scheme in Fig. 2 is slightly more general than that in Fig. 1 in the sense that it permits the use of oversampling (i.e., $M>D$ ). Notice that, when using oversampling, while the average rate of the available samples $\mathbf{y}_{d}(k), d=1, \ldots, D$, is $M / D T$, the samples $\hat{\mathbf{x}}(k)$ are still reconstructed at the desired rate $1 / T$. Therefore, this form of oversampling differs from the usual form in which the samples are reconstructed at a rate that is higher than the desired one. When $M>D$, the choice of filters $\mathbf{f}_{m}(z), m=1, \ldots, M$, which produce some given samples $\hat{\mathbf{x}}(k)$, is not unique. Hence, oversampling adds flexibility in the compensation design. This can be used to reduce the order of the compensation at the expense of a higher average sampling rate [17].

Using the scheme in Fig. 2, we will give in the following an overview of the available timing-mismatch compensation methods.

\section{A. EO Method [15]}

Yao and Thomas stated in [28] that a band-limited continuous-time signal $x(t)$, which is sampled using an irregular grid $\left\{t_{k}: k \in \mathbb{Z}\right\}$ with an average rate that is higher than or equal to the Nyquist rate, can be reconstructed using the formula

$$
x(t)=\sum_{k=-\infty}^{\infty} x\left(t_{k}\right) \frac{G(t)}{G^{\prime}\left(t_{k}\right)\left(t-t_{k}\right)}
$$

where

$$
G(t)=\left(t-t_{0}\right) \prod_{\substack{n=-\infty \\ n \neq 0}}^{\infty}\left(1-\frac{t}{t_{k}}\right)
$$

$G^{\prime}(t)$ is the derivative of $G(t)$, and the origin is shifted so that $t_{0}=0$.

It was shown in [15] that, if the sampling grid is periodic [i.e., it satisfies (2)] and the continuous-time signal needs only to be reconstructed on the regular grid $\left\{t_{k}=k T: k \in \mathcal{Z}\right\}$ (as in a time-interleaved ADC), then the reconstruction formula (4), (5) is equivalent to a filter-bank-based reconstruction, such as the one shown in Fig. 2. In the resulting scheme, $M=D$ and the filters $\mathbf{f}_{m}(z), m=1, \ldots, M$, are given by

$$
\mathbf{f}_{m}\left(e^{j \omega}\right)=\frac{1}{T} f_{m}\left(j \frac{\omega}{T}\right) e^{-j \frac{\omega t_{m}}{T}}, \quad|\omega|<\pi
$$

where $f_{m}(s)$ denotes the Laplace transform of

$$
f_{m}(t)=\operatorname{sinc}\left(\frac{\pi t}{M T}\right) \prod_{n=1, n \neq m}^{M} \frac{\sin \left(\frac{\pi\left(t+t_{m}-t_{n}\right)}{M T}\right)}{\sin \left(\frac{\pi\left(t_{m}-t_{n}\right)}{M T}\right)}
$$

evaluated at $s=j(\omega / T)$, and $\operatorname{sinc}(x)=\sin (x) / x$. Hence, the proposed scheme achieves perfect reconstruction if the filters $\mathbf{f}_{m}(z)$ are allowed to have infinite order and $x(t)$ is band limited to

$$
|f| \leq 1 / 2 T \text {. }
$$

\section{B. JL Method [16]}

Using the alias representation [29], the reconstructed samples $\hat{\mathbf{x}}(k)$ in Fig. 2 can be expressed in the frequency domain as

$$
\hat{\mathbf{x}}\left(e^{j \omega T}\right)=\sum_{d=-\infty}^{\infty} V_{d}(j \omega) x\left(j \omega-j \frac{2 \pi d}{D T}\right)
$$

with

$$
V_{d}(j \omega)=\frac{1}{D} \sum_{m=1}^{M} \mathbf{f}_{m}\left(e^{j \omega T}\right) e^{j\left(\omega-\frac{2 \pi d}{D T}\right) t_{m}} .
$$

In [16], the synthesis filters $\mathbf{f}_{m}(z)$ are designed using digital fractional delay filters. More precisely

$$
\mathbf{f}_{m}\left(e^{j \omega}\right)=a_{m} e^{-j \frac{\omega}{T} t_{m}}, \quad m=1, \ldots, M, \quad|\omega|<\pi
$$




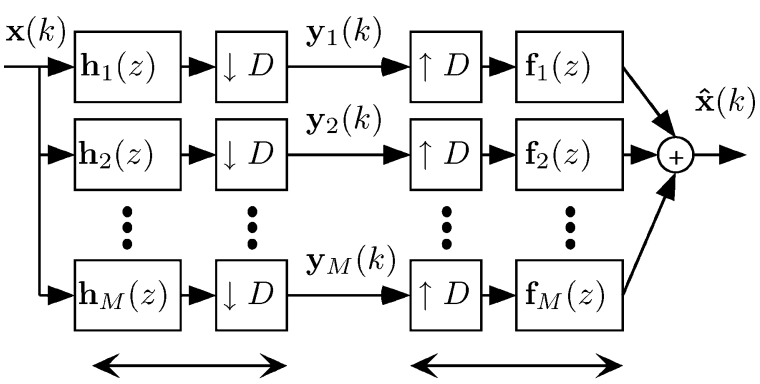

Discrete-time Equivalent Analysis Filterbank

Synthesis Filterbank

Fig. 3. Compensation scheme with discrete-time equivalent analysis filter bank.

so (6) becomes

$$
V_{d}(j \omega)=\frac{1}{D} \sum_{m=1}^{M} a_{m} e^{-j \frac{2 \pi d}{D T} t_{m}} .
$$

Then, the scaling coefficients $a_{m}, m=1, \ldots, M$, are chosen so that

$$
V_{d}(s)=0, \quad \forall-D_{0} \leq d \leq D_{0}
$$

with $D_{0}$ satisfying $M=2 D_{0}+1$. It follows that, if the filters $\mathbf{f}_{m}(z)$ are allowed to have infinite order (so that (7) is satisfied) and the input signal $x(t)$ is band limited to

$$
|f| \leq \frac{D_{0}+1}{2 T D}
$$

then (8) holds, and therefore, the proposed scheme achieves perfect reconstruction.

Remark 1: In view of (9), the order of the decay rate of the synthesis filters $\mathbf{f}_{m}(z)$ can be increased by requiring that (7) holds only within the band $|\omega|<\pi\left(D_{0}+1\right) / D$.

\section{PLH Method [17]}

If $x(t)$ is band limited to $|f| \leq 1 / 2 T$, we can write

$$
x(t)=\sum_{k=-\infty}^{\infty} x(k T) \operatorname{sinc}\left(\frac{t}{T}-k\right) .
$$

It is straightforward to see that the low branch in Fig. 2 can be replaced by the discrete-time equivalent scheme of Fig. 3, where the continuous-time signal $x(t)$ is replaced by its samples $\mathbf{x}(k)=x(k T)$, and the continuous-time filters $e^{t_{m} s}, m=$ $1, \ldots, M$, are replaced by the discrete-time filters $\mathbf{h}_{m}(z), m=$ $1, \ldots, M$ with the following frequency response:

$$
\mathbf{h}_{m}\left(e^{j \omega}\right)=e^{j \frac{\omega t_{m}}{T}}, \quad m=1, \ldots, M, \quad|\omega|<\pi .
$$

Now, using the polyphase representation [29], the scheme in Fig. 3 can be converted into that in Fig. 4, where the polyphase representations $\mathbf{X}(k)$ and $\hat{\mathbf{x}}(k)$ of $\mathbf{x}(k)$ and $\hat{\mathbf{x}}(k)$, respectively, are given by

$$
\begin{aligned}
& \mathbf{X}(k)=[\mathbf{x}(k D), \mathbf{x}(k D+1), \ldots, \mathbf{x}(k D+D-1)]^{\mathrm{T}} \\
& \hat{\mathbf{X}}(k)=[\hat{\mathbf{x}}(k D), \hat{\mathbf{x}}(k D+1), \ldots, \hat{\mathbf{x}}(k D+D-1)]^{\mathrm{T}}
\end{aligned}
$$

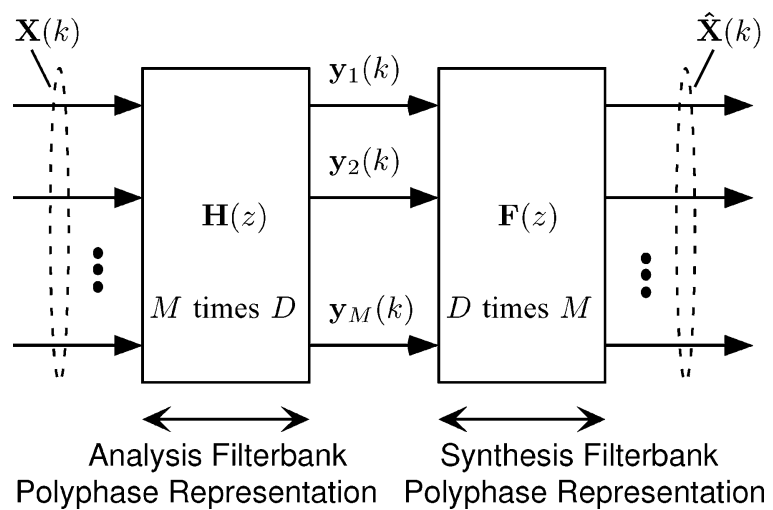

Fig. 4. Polyphase representation of Fig. 3.

with $\mathrm{T}$ denoting transposition. Also, the $(m, d)$ th entry $[\mathbf{H}(z)]_{m, d}$ of the polyphase representation $\mathbf{H}(z)$ of the analysis filter bank has impulse response

$$
[\mathbf{H}(k)]_{m, d}=\mathbf{h}_{m}(k D-d+1)
$$

and the $(d, m)$ th entry $[\mathbf{F}(z)]_{d, m}$ of the polyphase representation $\mathbf{F}(z)$ of the synthesis filter bank has impulse response

$$
[\mathbf{F}(k)]_{d, m}=\mathbf{f}_{m}(k D-d+1) .
$$

In view of Fig. 4, perfect reconstruction is guaranteed if

$$
\mathbf{F}(z) \mathbf{H}(z)=\mathbf{I}
$$

Then, for the given $a<b \in \mathbb{Z}$ defining the support of $\mathbf{F}(z)$ by $\mathbf{F}(k)=0: k \notin[a, b], \mathbf{F}(z)$ is designed as follows:

$$
\mathbf{F}(z)=\underset{\tilde{\mathbf{F}}(k)=0: k \notin[a, b]}{\arg \min } \frac{1}{2 \pi j} \oint_{|z|=1}\|\mathbf{I}-\tilde{\mathbf{F}}(z) \mathbf{H}(z)\|_{\mathrm{Fr}}^{2} \frac{\mathrm{d} z}{z}
$$

where $\|A\|_{\mathrm{Fr}}=\sqrt{\sum_{i=1}^{I} \sum_{j=1}^{J}\left|A_{i, j}\right|^{2}}$ denotes the Frobenius norm of the $I \times J$ matrix $A$ with entries $A_{i, j}$. Now, from (10) and (11), $\mathbf{H}(z)$ has infinite impulse response (IIR). Hence, in view of (13) and (12), the proposed scheme achieves perfect reconstruction if $M \geq D$, the filters $\mathbf{f}_{m}(z)$ are allowed to have infinite order, and $x(t)$ is band limited to

$$
|f| \leq 1 / 2 T .
$$

Remark 2: If $t_{m}$ is not an integer, the frequency response of $\mathbf{h}_{m}(z)$ in (10) has a discontinuity at $\omega=\pi$. This results in $\mathbf{H}(z)$ and the solution $\mathbf{F}(z)$ of (14) having very large impulse responses and, therefore, the synthesis filters $\mathbf{f}_{m}(z)$ having very high orders. Aiming at reducing the orders of $\mathbf{f}_{m}(z)$, a Kaiser window is used in [17] to increase the decay rate of the impulse responses of $\mathbf{h}_{m}(z), m=1, \ldots, M$.

Remark 3: Notice that, if $M=D$, there is only one choice of $\mathbf{F}(z)$ that satisfies (13). Hence, in this case, and if additionally $-a=b=\infty$, the Prendergast Levy Hurst (PLH) and Eldar Oppenheim (EO) methods are equivalent. 


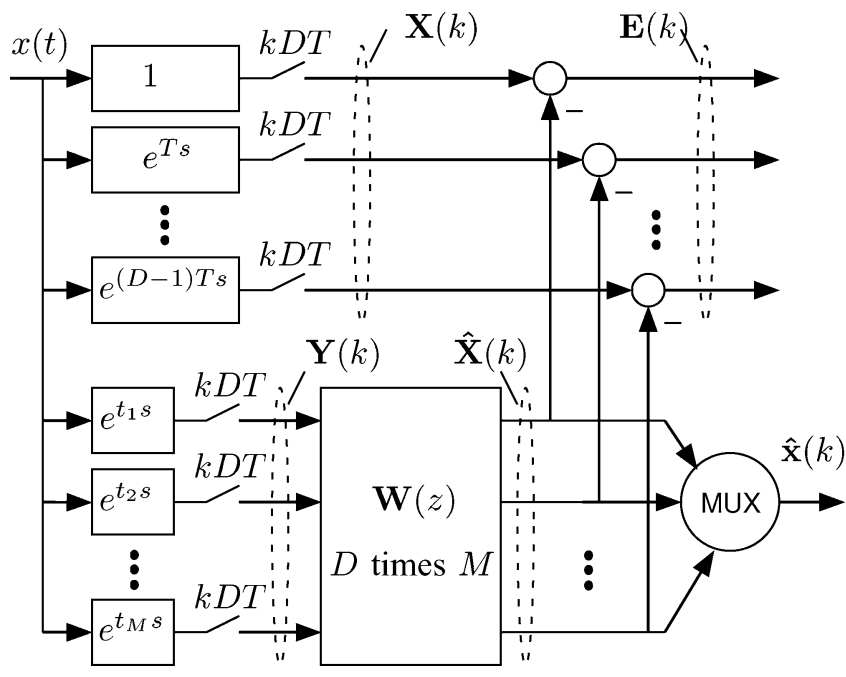

Fig. 5. Design scheme of the proposed compensation method.

\section{Proposed Timing-Mismatch Compensation Method}

The previously described EO, Johansson Lowenborg (JL), and PLH methods are designed under the assumption that the input signal is band limited. As mentioned in Section I, the drawback of this assumption is that it results in synthesis filters $\mathbf{f}_{m}(z)$ having very high orders. To go around this issue, in this section, we drop the band-limited assumption on the input signal, and we assume instead that it has a stationary known input spectrum (see the discussion in Section I about the practical implications of this assumption). We then design the compensation using a statistically optimal LMS criterion.

The measured sampling grid $\left\{t_{m}+k D T: k \in Z, m=\right.$ $1, \ldots, M\}$ is irregular and periodic with period $D T$, while the desired grid $\{k T: k \in Z\}$ is regular with rate $1 / T$. Now, both grids can be turned into regular sampling grids of rate $1 / D T$ taken on the vector signals

$$
\begin{aligned}
& Y(t)=\left[x\left(t_{1}+t\right), x\left(t_{2}+t\right), \ldots, x\left(t_{M}+t\right)\right]^{\mathrm{T}} \\
& X(t)=[x(t), x(T+t), \ldots, x((D-1) T+t)]^{\mathrm{T}}
\end{aligned}
$$

Hence, we can restate the problem as that of estimating $\mathbf{X}(k)=$ $X(k D T)$ from $\mathbf{Y}(k)=Y(k D T)$. We propose to do this estimation using a linear LMS criterion [21]. More precisely, the estimate $\hat{\mathbf{X}}(k)$ of $\mathbf{X}(k)$ is given by

$$
\hat{\mathbf{X}}(k)=\sum_{l \in \mathcal{I}} \mathbf{W}(l) \mathbf{Y}(k-l)
$$

where $\mathbf{W}(k)$ denotes the (matrix) impulse response of a filter, which is calculated to minimize the power of the reconstruction error signal $\mathbf{E}(k)=\mathbf{X}(k)-\hat{\mathbf{X}}(k)$, and $\mathcal{I}$ denotes the support of $\mathbf{W}(k)$. The idea is shown in Fig. 5.

Since $x(t)$ is assumed to be stationary, the signals $\mathbf{Y}(k)$ and $\mathbf{X}(k)$ are discrete-time stationary (vector) random processes, and therefore, $\mathbf{W}(k)$ can be found by solving

$$
\mathbf{W}=\underset{\mathbf{W}^{\prime}}{\arg \min } \mathcal{E}\left\{\left|\mathbf{X}(0)-\sum_{l \in \mathcal{I}} \mathbf{W}(l) \mathbf{Y}(-l)\right|^{2}\right\}
$$

where $\mathcal{E}\{\cdot\}$ denotes the expected value. Now, the solution of (15) requires that the estimation error is orthogonal to the the data used in the estimation, i.e.,

$$
\mathcal{E}\left\{\left(\mathbf{X}(0)-\sum_{l \in \mathcal{I}} \mathbf{W}(l) \mathbf{Y}(-l)\right) \mathbf{Y}^{T}(-k)\right\}=\mathbf{0}, \quad \forall k \in \mathcal{I}
$$

or, equivalently

$$
\mathbf{R}_{\mathbf{X Y}}(k)=\sum_{l \in \mathcal{I}} \mathbf{W}(l) \mathbf{R}_{\mathbf{Y}}(k-l), \quad \forall k \in \mathcal{I}
$$

where $\mathbf{R}_{\mathbf{Y}}(k)$ and $\mathbf{R}_{\mathbf{X Y}}(k)$ denote the correlation matrix of $\mathbf{Y}(k)$ and the cross-correlation matrix between $\mathbf{X}(k)$ and $\mathbf{Y}(k)$, respectively, i.e.,

$$
\begin{aligned}
\mathbf{R}_{\mathbf{Y}}(k) & =\mathcal{E}\left\{\mathbf{Y}(k) \mathbf{Y}^{T}(0)\right\} \\
\mathbf{R}_{\mathbf{X Y}}(k) & =\mathcal{E}\left\{\mathbf{X}(k) \mathbf{Y}^{T}(0)\right\} .
\end{aligned}
$$

To solve (16), we need to provide expressions for $\mathbf{R}_{\mathbf{Y}}(k)$ and $\mathbf{R}_{\mathbf{X Y}}(k)$. To do so, let $r_{x}(t)$ denote the autocorrelation of $x(t)$, i.e.,

$$
r_{x}(t)=\mathcal{E}\{x(t) x(0)\}
$$

Then, we have, for each $m, n=1, \ldots, M$

$$
\begin{aligned}
\mathbf{R}_{\mathbf{Y}}^{(m, n)}(k) & =\mathcal{E}\left\{\mathbf{Y}^{(m)}(k) \mathbf{Y}^{(n)}(0)\right\} \\
& =\mathcal{E}\left\{x\left(t_{m}+k M T\right) x\left(t_{n}\right)\right\} \\
& =r_{x}\left(k M T+t_{m}-t_{n}\right)
\end{aligned}
$$

and for each $d=1, \ldots, D$

$$
\begin{aligned}
\mathbf{R}_{\mathbf{X Y}}^{(d, m)}(k) & =\mathcal{E}\left\{\mathbf{Z}^{(d)}(k) \mathbf{Y}^{(m)}(0)\right\} \\
& =\mathcal{E}\left\{x((d-1) T+k D T) x\left(t_{m}\right)\right\} \\
& =r_{x}\left((k D+d-1) T-t_{m}\right) .
\end{aligned}
$$

The solution of (16) depends on the support $\mathcal{I}$ of the impulse response $\mathbf{W}(k)$. We solve (16) in the following by considering two cases. In Section IV-A, we consider the case $\mathcal{I}=\mathbb{Z}$, which is known as the Wiener smoother in the context of statistical signal processing [21], [22]. This is obviously the best possible solution, but it yields a noncausal IIR filter $\mathbf{W}(z)$. In a practical implementation, the support $\mathcal{I}$ needs to be truncated. This can be done by simply truncating the support of the Wiener smoother, but this yields an unoptimal solution. We do this in an optimal way in Section IV-B.

\section{A. Unconstrained Case (IIR-LMS Method)}

As mentioned earlier, if $\mathcal{I}=\mathbb{Z}$, the solution of (16) is known as the Wiener smoother, and its derivation can be found in any standard book on statistical signal processing (e.g., [21, Sec. 7.8]). The solution is given by

$$
\mathbf{W}(z)=\mathbf{S}_{\mathbf{X Y}}(z) \mathbf{S}_{\mathbf{Y}}^{\dagger}(z)
$$

where

$$
\mathbf{S}_{\mathbf{Y}}=\mathcal{Z}\left\{\mathbf{R}_{\mathbf{Y}}\right\} \quad \text { and } \quad \mathbf{S}_{\mathbf{X Y}}=\mathcal{Z}\left\{\mathbf{R}_{\mathbf{X Y}}\right\}
$$

and $\mathbf{S}_{\mathbf{Y}}^{\dagger}(z)$ denotes the (Moore-Penrose) pseudoinverse [30] of the matrix $\mathbf{S}_{\mathbf{Y}}(z)$. 


\section{B. Constrained Case (FIR-LMS Method)}

Let $\mathcal{I}=[a, b]$, with $a<b \in \mathbb{Z}$ and $c=b-a+1$. Also, let $\mathbf{R}_{\mathbf{X Y}}^{(d,:)}$ and $\mathbf{W}^{(d,:)}$ denote the $d$-rows of $\mathbf{R}_{\mathbf{X Y}}$ and $\mathbf{W}$, respectively. Then, we can write (16) as

$$
\mathbf{R}_{\mathbf{X Y}}^{(d,:)}(k)=\sum_{l=a}^{b} \mathbf{W}^{(d,:)}(l) \mathbf{R}_{\mathbf{Y}}(k-l)
$$

for all $a \leq k \leq b$ and $1 \leq d \leq D$. Define the matrix $\underline{\mathbf{R}_{\mathbf{Y}}} \in$ $\mathbb{R}^{c M \times c M}$ by

$$
\underline{\mathbf{R}_{\mathbf{Y}}}=\left[\begin{array}{ccc}
\underline{\mathbf{R}_{\mathbf{Y}}^{(1,1)}} & \cdots & \underline{\mathbf{R}_{\mathbf{Y}}^{(1, M)}} \\
\vdots & \ddots & \vdots \\
\mathbf{R}_{\mathbf{Y}}^{(M, 1)} & \cdots & \underline{\mathbf{R}_{\mathbf{Y}}^{(M, M)}}
\end{array}\right]
$$

where, for each $1 \leq m, n \leq M$, the submatrix $\mathbf{R}_{\mathbf{Y}}^{(m, n)} \in \mathbb{R}^{c \times c}$ is given by

$$
\left(\underline{\mathbf{R}_{\mathbf{Y}}^{(m, n)}}\right)^{(i, j)}=\mathbf{R}_{\mathbf{Y}}^{(m, n)}(j-i)=r_{x}\left((j-i) M T+t_{m}-t_{n}\right)
$$

for all $1 \leq i, j \leq c$. Also, for each $1 \leq d \leq D$, define the row vectors $\underline{\mathbf{R}_{\mathbf{X Y}}^{(d,:)}} \in \mathbb{R}^{c M}$ and $\underline{\mathbf{W}^{(d,:)}} \in \mathbb{R}^{c M}$ by

$$
\begin{aligned}
\underline{\mathbf{R}_{\mathbf{X Y}}^{(d,:)}} & =\left[\underline{\mathbf{R}_{\mathbf{X Y}}^{(d, 1)}}, \ldots, \underline{\mathbf{R}_{\mathbf{X Y}}^{(d, M)}}\right] \\
\underline{\mathbf{W}^{(d,:)}} & =\left[\underline{\mathbf{W}_{\mathbf{X Y}}^{(d, 1)}}, \ldots, \underline{\mathbf{W}_{\mathbf{X Y}}^{(d, M)}}\right]
\end{aligned}
$$

where, for each $1 \leq d \leq D$ and $1 \leq m \leq M$, the subvectors

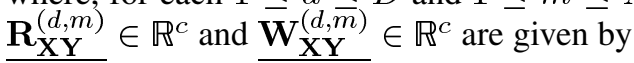

$$
\begin{aligned}
& \underline{\mathbf{R}_{\mathbf{X Y}}^{(d, m)}}=\left[\mathbf{R}_{\mathbf{X Y}}^{(d, m)}(a), \ldots, \mathbf{R}_{\mathbf{X Y}}^{(d, m)}(b)\right] \\
& =\left[r_{x}\left(a D T+(d-1) T-t_{1}\right), \ldots,\right. \\
& \left.r_{x}\left(b D T+(d-1) T-t_{M}\right)\right] \\
& \underline{\mathbf{W}_{\mathbf{X Y}}^{(d, m)}}=\left[\mathbf{W}^{(d, m)}(a), \ldots, \mathbf{W}^{(d, m)}(b)\right]
\end{aligned}
$$

Then, (20) can be written as

$$
\underline{\mathbf{R}_{\mathbf{X Y}}^{(d,:)}}=\underline{\mathbf{W}^{(d,:)}} \underline{\mathbf{R}_{\mathbf{Y}}}, \quad \forall 1 \leq d \leq D .
$$

Now, defining the matrices $\underline{\mathbf{R}_{\mathbf{X Y}}} \in \mathbb{R}^{D \times c M}$ and $\underline{\mathbf{W}} \in \mathbb{R}^{D \times c M}$ by

$$
\underline{\mathbf{R}_{\mathbf{X Y}}}=\left[\begin{array}{c}
\frac{\mathbf{R}_{\mathbf{X Y}}^{(1,:)}}{\vdots} \\
\vdots \\
\mathbf{R}_{\mathbf{X Y}}^{(D,:)}
\end{array}\right] \quad \underline{\mathbf{W}}=\left[\begin{array}{c}
\underline{\mathbf{W}^{(1,:)}} \\
\vdots \\
\underline{\mathbf{W}^{(D,:)}}
\end{array}\right]
$$

the solution of (16) is obtained as follows:

$$
\underline{\mathbf{W}}=\underline{\mathbf{R}}_{\mathbf{X Y}} \mathbf{R}_{\mathbf{Y}}^{\dagger}
$$

\section{Interpretation as a Filter-Bank-Based Compensation Method}

As mentioned in Section I, the proposed compensation method is equivalent to a filter-bank-based method, such as the one shown in Fig. 2. To see this, in view of (12), we can interpret the $D \times M$ transfer matrix $\mathbf{W}(z)$ as the polyphase representation of a synthesis filter bank with upsampling factor $D$ and filters

$$
\mathbf{f}_{m}(z)=\sum_{d=0}^{D-1} z^{-d}\left[\mathbf{W}\left(z^{D}\right)\right]_{d, m}, \quad m=1, \ldots, M
$$

where $[\mathbf{W}(z)]_{d, m}$ denotes the $(d, m)$ th entry of the transfer matrix $\mathbf{W}(z)$.

\section{BAND-LIMITED CASE}

A common feature of the methods in Section III is that they achieve perfect reconstruction if $x(t)$ is band limited and that the order of the compensator is not restricted. In this section, we show that, under the same conditions, and if $M \geq D$, the proposed IIR-LMS design is independent of the input spectrum and that it also enjoys the perfect-reconstruction property. Additionally, we show that, if $x(t)$ has a flat spectrum within its passband, then the proposed LMS method is equivalent to the PLH method, even when the order of the compensator is restricted (i.e., in the FIR case). Furthermore, in view of Remark 3, this implies that, if the compensator order is not restricted (i.e., in the IIR case), all three methods, namely, EO, PLH, and the proposed LMS, are equivalent.

As described in Section IV-C, the proposed method can represented as in Fig. 2. If $x(t)$ is band limited with support in $|f|<1 / 2 T$, as explained in Section III-C, we can represent Fig. 2 in its polyphase form shown in Fig. 4. It follows that $\mathbf{Y}(k)=\sum_{l=-\infty}^{\infty} \mathbf{H}(l) \mathbf{X}(k-l)$, and therefore

$$
\begin{aligned}
\mathbf{S}_{\mathbf{Y}}(z) & =\mathbf{H}(z) \mathbf{S}_{\mathbf{X}}(z) \mathbf{H}^{*}(z) \\
\mathbf{S}_{\mathbf{X Y}}(z) & =\mathbf{S}_{\mathbf{X}}(z) \mathbf{H}^{*}(z)
\end{aligned}
$$

where $\mathbf{H}^{*}(t)=\mathbf{H}^{T}(-t)$. We will use (22) and (23) in the following to show the aforementioned properties.

\section{A. IIR-LMS Design Is Independent of the Input Spectrum}

Equations (4) and (5) imply that the matrix $\mathbf{H}(z)$ has a left inverse on the unit circle (i.e., there exists $\mathbf{H}^{L}(z)$ such that $\mathbf{H}^{L}(z) \mathbf{H}(z)=\mathbf{I}$ for all $\left.|z|=1\right)$. This, in turn, implies that

$$
\mathbf{H}^{\dagger}(z) \mathbf{H}(z)=\mathbf{I} \forall|z|=1
$$

and for all $\mathbf{U}(z)$

$$
(\mathbf{H}(z) \mathbf{U}(z))^{\dagger}=\mathbf{U}^{\dagger}(z) \mathbf{H}^{\dagger}(z), \quad \forall|z|=1 .
$$

From (22) and (25), it follows that

$$
\mathbf{S}_{\mathbf{Y}}^{\dagger}(z)=\left(\mathbf{H}(z) \mathbf{S}_{\mathbf{X}}(z) \mathbf{H}^{*}(z)\right)^{\dagger}=\mathbf{H}^{\dagger *}(z) \mathbf{S}_{\mathbf{X}}^{\dagger}(z) \mathbf{H}^{\dagger}(z) .
$$

Then, putting (26) and (23) into (19) and using (24), we obtain

$$
\begin{aligned}
\mathbf{W}(z) & =\mathbf{S}_{\mathbf{X}}(z) \mathbf{H}^{*}(z) \mathbf{H}^{\dagger *}(z) \mathbf{S}_{\mathbf{X}}^{\dagger}(z) \mathbf{H}^{\dagger}(z) \\
& =\mathbf{S}_{\mathbf{X}}(z) \mathbf{S}_{\mathbf{X}}^{\dagger}(z) \mathbf{H}^{\dagger}(z)
\end{aligned}
$$

which, under the mild assumption that $\mathbf{S}_{\mathbf{X}}(z)$ is nonsingular almost everywhere on the unit circle, results in

$$
\mathbf{W}(z)=\mathbf{H}^{\dagger}(z)
$$

which is independent of the input spectrum. 


\section{B. IIR-LMS Design Achieves Perfect Reconstruction}

We need the following lemma.

Lemma 1: If $x(t)$ is band limited with support in $|f|<1 / T$ and $M \geq D$, then

$$
\mathbf{S}_{\mathbf{X Y}}(z) \mathbf{S}_{\mathbf{Y}}^{\dagger}(z) \mathbf{S}_{\mathbf{Y X}}(z)=\mathbf{S}_{\mathbf{X}}(z)
$$

Proof: Combining (23), (26), and (24), we have

$$
\mathbf{S}_{\mathbf{X Y}}(z) \mathbf{S}_{\mathbf{Y}}^{\dagger}(z) \mathbf{S}_{\mathbf{Y X}}(z)=\mathbf{S}_{\mathbf{X}}(z) \mathbf{S}_{\mathbf{X}}^{\dagger}(z) \mathbf{S}_{\mathbf{X}}(z)=\mathbf{S}_{\mathbf{X}}(z)
$$

where the second equality follows from a property of pseudoinverses [30].

The $z$-transform $\mathbf{S}_{\mathbf{E}}(z)$ of the autocorrelation matrix $\mathbf{R}_{\mathbf{E}}$ of the error signal $\mathbf{E}(k)=\mathbf{X}(k)-\hat{\mathbf{X}}(k)$ is given by

$$
\begin{array}{r}
\mathbf{S}_{\mathbf{E}}(z)=\mathbf{S}_{\mathbf{X}}(z)-\mathbf{S}_{\mathbf{X Y}}(z) \mathbf{W}^{*}(z)-\mathbf{W}(z) \mathbf{S}_{\mathbf{Y X}}(z) \\
+\mathbf{W}(z) \mathbf{S}_{\mathbf{Y}}(z) \mathbf{W}^{*}(z)
\end{array}
$$

Now, using (19) and Lemma 1, we have

$$
\mathbf{W}(z) \mathbf{S}_{\mathbf{Y X}}(z)=\mathbf{S}_{\mathbf{X Y}}(z) \mathbf{S}_{\mathbf{Y}}^{\dagger}(z) \mathbf{S}_{\mathbf{Y X}}(z)=\mathbf{S}_{\mathbf{X}}(z)
$$

and in a similar way

$$
\mathbf{S}_{\mathbf{X Y}}(z) \mathbf{W}^{*}(z)=\mathbf{S}_{\mathbf{X}}(z)
$$

Also, we have

$$
\begin{aligned}
\mathbf{W}(z) \mathbf{S}_{\mathbf{Y}}(z) \mathbf{W}^{*}(z) & =\mathbf{S}_{\mathbf{X Y}}(z) \mathbf{S}_{\mathbf{Y}}^{\dagger}(z) \mathbf{S}_{\mathbf{Y}}(z) \mathbf{S}_{\mathbf{Y}}^{\dagger}(z) \mathbf{S}_{\mathbf{Y X}}(z) \\
& =\mathbf{S}_{\mathbf{X Y}}(z) \mathbf{S}_{\mathbf{Y}}^{\dagger}(z) \mathbf{S}_{\mathbf{Y X}}(z) \\
& =\mathbf{S}_{\mathbf{X}}(z)
\end{aligned}
$$

where the second equality follows from a property of pseudoinverses [30] and the third one follows from Lemma 1. Finally, putting (28)-(30) in (27), we have

$$
\mathbf{S}_{\mathbf{E}}(z)=\mathbf{S}_{\mathbf{X}}(z)-\mathbf{S}_{\mathbf{X}}(z)-\mathbf{S}_{\mathbf{X}}(z)+\mathbf{S}_{\mathbf{X}}(z)=0
$$

implying that the proposed compensation achieves perfect reconstruction.

\section{Equivalence Between the PLH and LMS Methods Under a Flat Band-Limited Input Spectrum}

Let the input power spectrum be given by

$$
\phi_{x}(j \omega)= \begin{cases}1, & |\omega|<\frac{\pi}{T} \\ 0, & \text { otherwise. }\end{cases}
$$

Then, $\mathbf{S}_{\mathbf{Z}}(z)=\mathbf{I}$, and from (22) and (23), the LMS design (16) can be written as

$$
\mathbf{H}^{*}(k)=\sum_{l \in \mathcal{I}} \mathbf{W}(l) \mathbf{H H}^{*}(k-l), \quad \forall k \in \mathcal{I}
$$

where, with some abuse of notation, $\mathbf{H}^{*}(k)$ and $\mathbf{H H}^{*}(k)$ denote the inverse $z$-transforms of $\mathbf{H}^{*}(z)$ and $\mathbf{H}(z) \mathbf{H}^{*}(z)$, respectively. Now, the design (14) for the PLH method can be written as

$$
\mathbf{F}=\underset{\tilde{\mathbf{F}}(k)=0: k \notin \mathcal{I}}{\arg \min }\|\mathbf{I}-\tilde{\mathbf{F}} \mathbf{H}\|
$$

where $\mathcal{I}=[a, b]$, the norm $\|\mathbf{U}\|=\langle\mathbf{U}, \mathbf{U}\rangle^{1 / 2}$, and

$$
\begin{aligned}
\langle\mathbf{U}, \mathbf{V}\rangle & =\frac{1}{2 \pi j} \oint_{|z|=1} \operatorname{Tr}\left\{\mathbf{U}(z) \mathbf{V}^{*}(z)\right\} \frac{\mathrm{d} z}{z} \\
& =\sum_{k \in \mathbb{Z}} \operatorname{Tr}\left\{\sum_{l \in \mathcal{I}} \mathbf{U}(l) \mathbf{V}^{*}(k-l)\right\}
\end{aligned}
$$

with $\operatorname{Tr}\{A\}=\sum_{i=1}^{I} A_{i, i}$ being the trace operator of the $I \times$ $I$ matrix $A$ with entries $A_{i, j}$, and $\mathbf{V}^{*}(k)$ denoting the inverse $z$-transforms of $\mathbf{V}^{*}(z)$. Then, we say that (33) is satisfied if and only if, for all $\tilde{\mathbf{F}}(z)$ such that $\tilde{\mathbf{F}}(k)=0: k \notin \mathcal{I}$

$$
\begin{aligned}
0 & =\langle\mathbf{I}-\mathbf{F H}, \tilde{\mathbf{F}} \mathbf{H}\rangle \\
& =\frac{1}{2 \pi j} \oint_{|z|=1} \operatorname{Tr}\left\{(\mathbf{I}-\mathbf{F}(z) \mathbf{H}(z)) \mathbf{H}^{*}(z) \tilde{\mathbf{F}}^{*}(z)\right\} \frac{\mathrm{d} z}{z} \\
& =\frac{1}{2 \pi j} \oint_{|z|=1} \operatorname{Tr}\left\{\left(\mathbf{H}^{*}(z)-\mathbf{F}(z) \mathbf{H}(z) \mathbf{H}^{*}(z)\right) \tilde{\mathbf{F}}^{*}(z)\right\} \frac{\mathrm{d} z}{z} \\
& =\left\langle\mathbf{H}^{*}-\mathbf{F} \mathbf{H H}^{*}, \tilde{\mathbf{F}}\right\rangle .
\end{aligned}
$$

In view of (34), this implies that

$$
\sum_{k \in \mathbb{Z}} \operatorname{Tr}\left\{\left(\mathbf{H}^{*}(k)-\sum_{l \in \mathcal{I}} \mathbf{F}(l) \mathbf{H H}^{*}(k-l)\right) \tilde{\mathbf{F}}^{T}(k)\right\}=0
$$

and comparing with (32), it follows that the PLH and LMS methods are equivalent for any support $\mathcal{I}$.

\section{Simulations}

In order to evaluate the proposed compensation method, we compare its performance with those of the EO, JL, and PLH methods described in Section III. To this end, we consider the example used in [16] and [17], which uses five-channel $\operatorname{ADCs}(M=5)$ with no oversampling $(D=5)$ and with sampling phases $[0,0.96 T, 2.02 T, 2.99 T, 4.03 T]$. This corresponds to the following timing mismatches $[0,-0.04 T, 0.02 T,-0.01 T, 0.03 T]$. For simplicity, the sampling period is $T=1$. In order to quantify the performance, we use the inverse of the SINAD, i.e.,

$$
S I N A D^{-1}=10 \log _{10}\left(\frac{\sum_{k=1}^{K}|\mathbf{x}(k)-\hat{\mathbf{x}}(k)|^{2}}{\sum_{k=1}^{K}|\mathbf{x}(k)|^{2}}\right) .
$$

\section{A. Known Input Spectrum}

In this section, we generate the input signal using a random process (instead of a sum of sinusoids, as done in [16] and [17]). The filters $\mathbf{f}_{m}(z), m=1, \ldots, M$, in the EO, JL, and PLH methods have, in theory, infinite order and therefore need to be truncated. In the first simulation, we truncate the impulse responses $\mathbf{f}_{m}(k), m=1, \ldots, M$, of the EO, JL, and PLH methods to 1500 taps. Also, we design the FIR-LMS method using $a=-149$ and $b=150$ so that the equivalent synthesis filter bank (21) has the same number (1500) of taps. We generate the input signal as filtered white noise using a Butterworth low-pass filter (input filter) of 20th order and cutoff frequency 


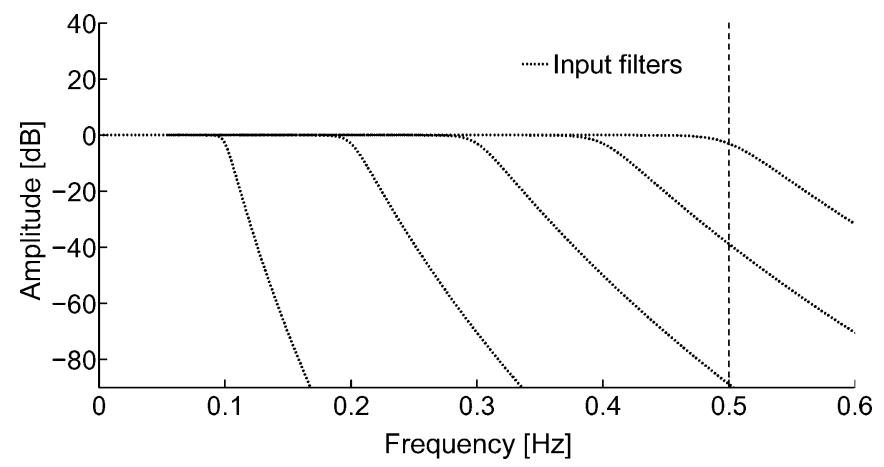

Fig. 6. Frequency response of a family of 20th-order Butterworth low-pass filter with different cutoff-frequency values.

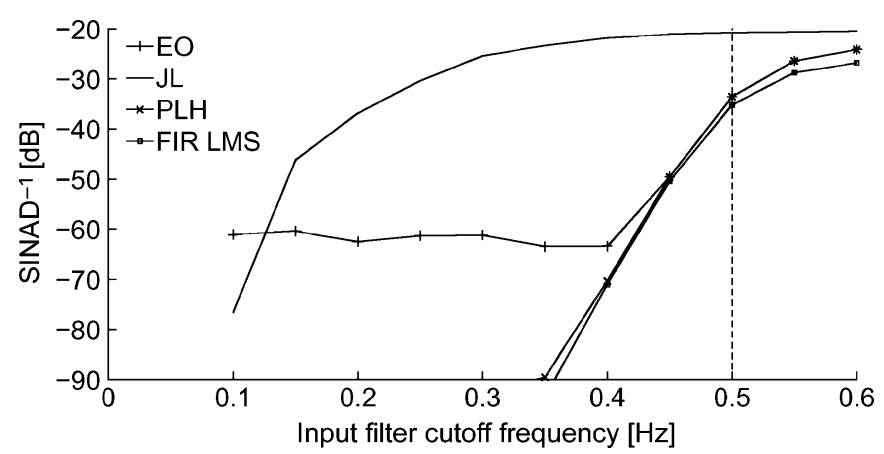

Fig. 7. Performance comparison of the different compensation methods with a compensator's complexity constraint of 1500 mult./sample.

$\omega_{c}$. The frequency response of a family of such filters with different values of $\omega_{c}$ is shown in Fig. 6. We compare the performances of the different methods for several values of $\omega_{c}$. The result is shown in Fig. 7. We see that, while the FIR-LMS method outperforms the other methods, the PLH method still performs satisfactorily over the whole cutoff-frequency range.

Notice that, for large cutoff-frequency values, the performance of all methods is limited by the amount of signal power above the Nyquist frequency, so all methods tend to perform similarly. However, the FIR-LMS method performs slightly better due to its optimal nature. Also, the performance of the different methods improve as the cutoff-frequency value decreases, until reaching a given threshold level of $S I N A D^{-1}$, from where the performance remains roughly constant (this is better seen in Figs. 8 and 9 in the following). This threshold results from the truncation of the synthesis filters $\mathbf{f}_{m}(k)$, $m=1, \ldots, M$, and its level can be decreased by increasing the truncation order.

The performance of the JL method is rather poor in the high cutoff-frequency range. One reason for this is that, according to (9), the method is suitable for signals with cutoff frequency that is smaller than $0.3 \mathrm{~Hz}$ (instead of $0.5 \mathrm{~Hz}$ as for the EO and PLH methods). However, notice that, even if we shift its performance curve by $0.2 \mathrm{~Hz}$ to the right, its performance is still inferior to that of the other methods. The reason for this is that the choice of fractional delays for the synthesis filter bank (7) is not optimal. On the other hand, as mentioned in Section I, this choice has the advantage of permitting an efficient redesign when the sampling pattern changes.

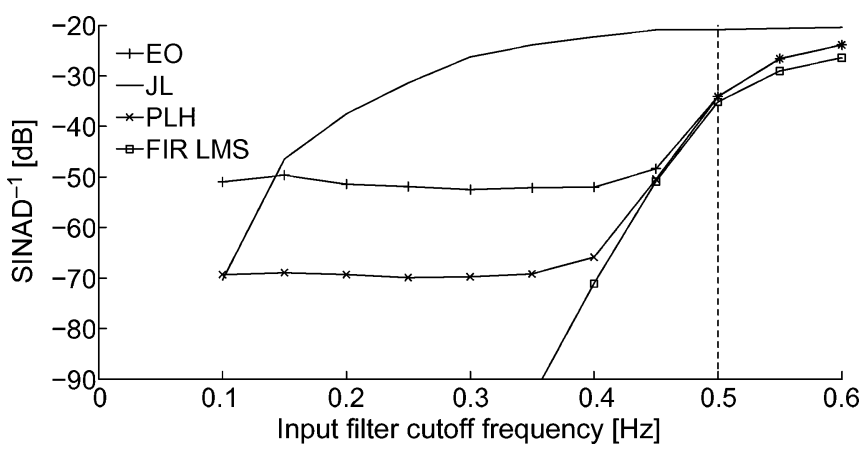

Fig. 8. Performance comparison of the different compensation methods with a compensator's complexity constraint of 150 mult./sample.

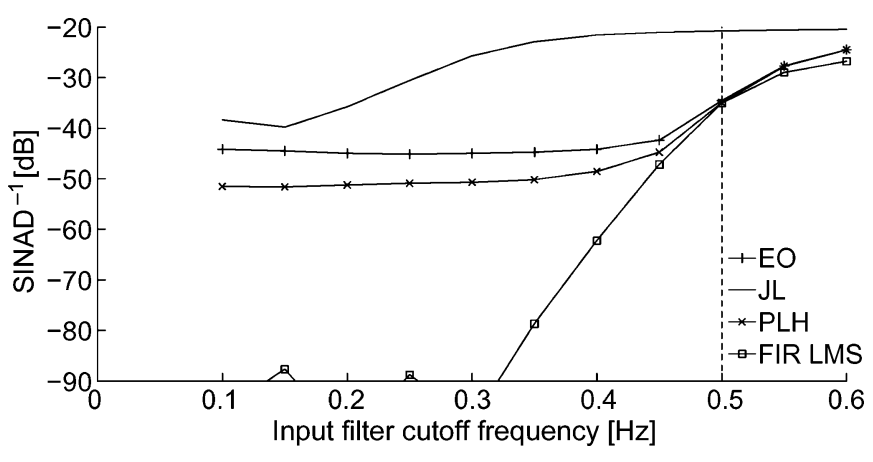

Fig. 9. Performance comparison of the different compensation methods with a compensator's complexity constraint of 15 mult./sample.

In the second simulation, following the design in [17], we truncate the impulse responses $\mathbf{f}_{m}(k), m=1, \ldots, M$, of the EO, JL, and PLH methods to 150 taps, and we use $a=-14$ and $b=15$ for the FIR-LMS method so that the equivalent synthesis filter bank has the same number (150) of taps. We generate the input signal as in the first simulation, and we show the result in Fig. 8. We see that the FIR-LMS method clearly outperforms the other methods for low cutoff-frequency values.

In the third simulation, we consider a very low compensation complexity. We truncate the impulse responses $\mathbf{f}_{m}(k), m=$ $1, \ldots, M$, to 15 taps, and we use the FIR-LMS method with $a=-1$ and $b=1$ so that the equivalent synthesis filter bank has 15 taps. The result is shown in Fig. 9. We see that, while the performance of the EO, JL, and PLH methods has clearly degraded, the FIR-LMS method is still able to achieve an average $S I N A D^{-1}$ of about $-90 \mathrm{~dB}$ for low cutoff-frequency values.

\section{B. Unknown Input Spectrum}

The design of the LMS method is optimum for a given power spectrum $\phi_{x}=\mathcal{F}\left\{r_{x}\right\}$ of the input signal $x(t)$. In this section, we evaluate the performance degradation of the LMS method when there is a mismatch between the input-signal power spectrum, determined by the input filter, and the nominal power spectrum used to design the LMS compensator, determined by a nominal filter. We use a compensator order of 150 taps for all methods, and a fixed nominal filter designed as a Butterworth low-pass filter of tenth order and cutoff frequency of $0.4 \mathrm{~Hz}$. We use the same input filter used in Section VI-A, i.e., a Butterworth low-pass input filter of 20th order and variable cutoff (angular) frequency $\omega_{c}$. The frequency responses of the nominal 


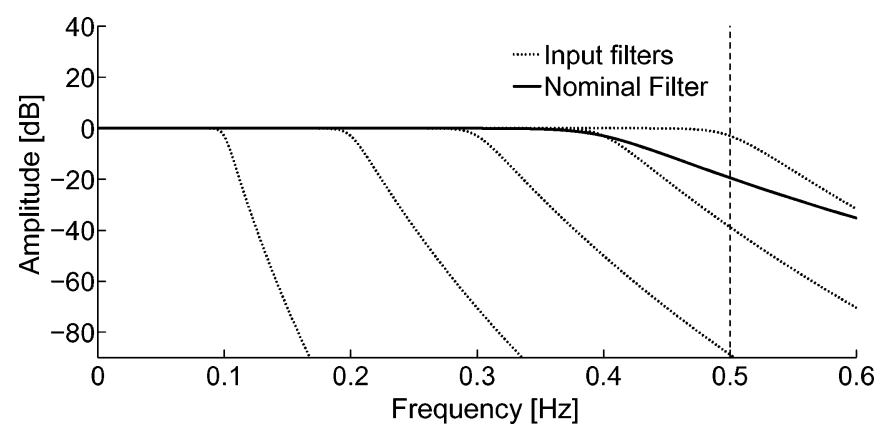

Fig. 10. Frequency response of the input filters with different cutoff-frequency values and the nominal filter used to design the LMS method.

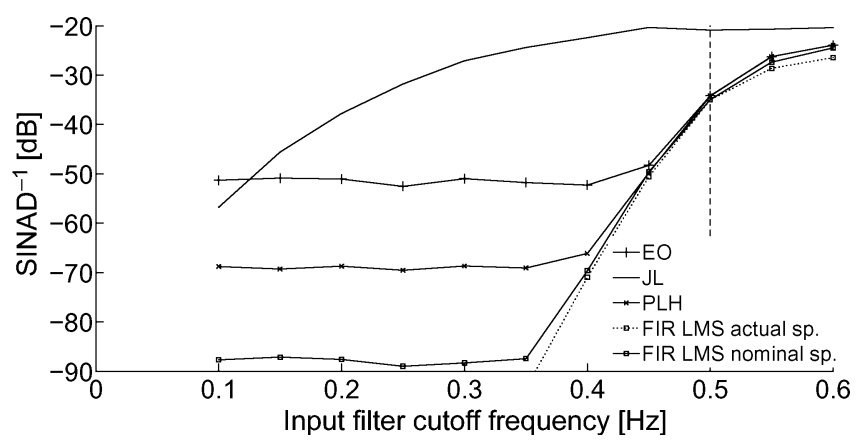

Fig. 11. Performance comparison of the different compensation methods with input-power-spectrum mismatch (complexity constraint = 150 mult./sample).

TABLE I

PERFORMANCE COMPARISON OF THE DIFFERENT COMPENSATION METHODS WHEN $x(t)$ IS A MIXTURE OF SINUSOIDS(complexity constraint $=$ 150 mult./sample).

\begin{tabular}{|c||c|c|c|c|}
\hline Method & EO & JL & PLH & FIR-LMS \\
\hline$S I N A D^{-1}$ & $-58.37 \mathrm{~dB}$ & $-30.00 \mathrm{~dB}$ & $-71.65 \mathrm{~dB}$ & $-172.83 \mathrm{~dB}$ \\
\hline
\end{tabular}

and input filters are shown in Fig. 10, and the simulation result is shown in Fig. 11, where we have also included the performance obtained with the LMS method without spectral mismatch (i.e., when using the actual input power spectrum). We see that, while losing some performance due to the spectral mismatch, the LMS method clearly outperform the EO, JL, and PLH methods in the low cutoff-frequency range.

\section{Periodic Input Signal}

In this section, we evaluate the performance of the different methods when using a periodic input signal, as done in [16] and [17]. We consider the same example used in those works, i.e., the input signal is given by

$$
x(t)=\sum_{k=1}^{4} \sin \left(2 \pi f_{k} t+\phi_{k}\right)
$$

where $f_{k}=k / 16$ and the phases $\phi_{k}, k=1,2,3,4$ are random variables uniformly distributed in the interval $(0,2 \pi]$. We use a compensator order of 150 taps for all methods, and a Butterworth low-pass input filter of 20th order and cutoff frequency of $0.4 \mathrm{~Hz}$ for the design of the FIR-LMS method. The resulting $S I N A D^{-1}$ values are given in Table I, showing the clear advantage of the FIR-LMS method.

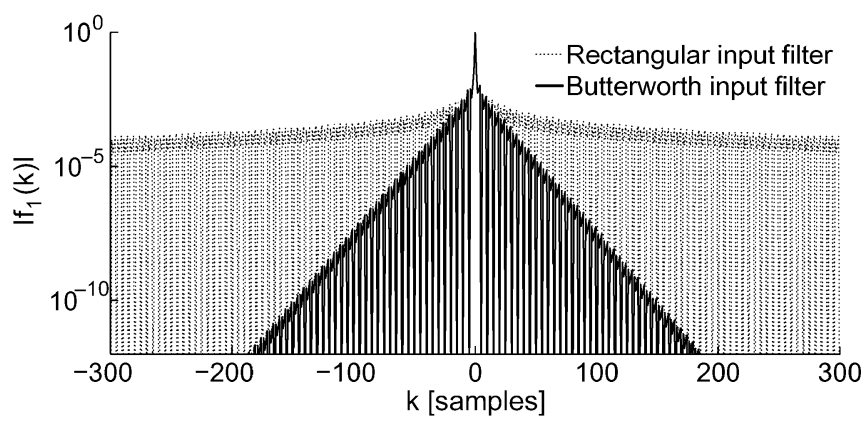

Fig. 12. Comparison of the synthesis filter banks obtained using rectangular and Butterworth input filters.

\section{Band-Limited Versus Non-Band-Limited Input Spectrum}

As mentioned previously, the computational advantage of the proposed LMS method comes from the fact that doing a band-limited assumption on the input spectrum results in synthesis filters $\mathbf{f}_{m}(k), m=1, \ldots, M$, with a very slow decay rate. To illustrate this point, we compare in Fig. 12 the magnitude of the impulse response of the first channel synthesis filter $\mathbf{f}_{1}(z)$, which is obtained under two different assumptions on the input spectrum. The first is the band-limited assumption to the band $|f|<1 / 2 T$. As pointed out in Section $\mathrm{V}-\mathrm{A}$, the resulting synthesis filters are independent of the shape of the input spectrum within the band $|f|<1 / 2 T$, and as shown in Section V-C, they equal those obtained using the PLH method. The second assumption is that the input signal is filtered white noise, using a Butterworth low-pass input filter of 20th order and cutoff frequency of $0.4 \mathrm{~Hz}$. Fig. 12 clearly shows the faster decay rate obtained when the band-limited assumption is dropped.

\section{CONCLUSION}

We have proposed a compensation method for timing mismatches in time-interleaved ADCs. As opposite to other approaches, the proposed method does not assume that the input signal is band limited but assumes instead that it has a known stationary input power spectrum. The compensation is then designed in a statistically optimal sense. This largely reduces the compensation order, and thus computing complexity, required to achieve a given reconstruction accuracy. If the input signal is not assumed to be band limited, perfect reconstruction cannot be guaranteed. However, we have shown that, under the band-limited assumption, the proposed method achieves perfect reconstruction if no constraints are imposed on the order of the compensation. Simulation results show that the proposed compensation method clearly outperforms the other methods, in terms of the SINAD, for a given fixed compensation order. We have also shown that a rough estimate of the input spectrum can be used without much performance loss, showing that an accurate knowledge of the input spectrum is not necessarily needed.

\section{ACKNOWLEDGMENT}

This work was developed in part in the Numerical Harmonic Analysis Group, Faculty of Mathematics, University of Vienna, Vienna, Austria. 


\section{REFERENCES}

[1] W. C. J. Black and D. A. Hodges, "Time interleaved converter arrays," IEEE J. Solid-State Circuits, vol. SSC-15, no. 6, pp. 1022-1029, Dec. 1980.

[2] K. Poulton, R. Neff, B. Setterberg, B. Wuppermann, T. Kopley, R. Jewett, J. Pernillo, C. Tan, and A. Montijo, "A 20 GS/s 8 b ADC with a $1 \mathrm{MB}$ memory in $0.18 \mu \mathrm{m}$ CMOS," in Proc. IEEE Int. Solid-State Circuits Conf. Dig. Tech. Papers, 2003, pp. 317-319.

[3] C. Vogel, "The impact of combined channel mismatch effects in timeinterleaved ADCs," IEEE Trans. Instrum. Meas., vol. 54, no. 1, pp. 415-427, Feb. 2005.

[4] S.-W. Sin, U.-F. Chio, S.-P. U, and R. Martins, "Statistical spectra and distortion analysis of time-interleaved sampling bandwidth mismatch," IEEE Trans. Circuits Syst. II, Exp. Briefs, vol. 55, no. 7, pp. 648-652, Jul. 2008.

[5] H. Jin and E. Lee, "A digital-background calibration technique for minimizing timing-error effects in time-interleaved ADCs," IEEE Trans. Circuits Syst. II, Analog Digit. Signal Process., vol. 47, no. 7, pp. 603-613, Jul. 2000.

[6] Y.-C. Jenq, "Digital spectra of nonuniformly sampled signals: A robust sampling time offset estimation algorithm for ultra high-speed waveform digitizers using interleaving," IEEE Trans. Instrum. Meas., vol. 39, no. 1, pp. 71-75, Feb. 1990.

[7] S. Jamal, D. Fu, M. Singh, P. Hurst, and S. Lewis, "Calibration of sample-time error in a two-channel time-interleaved analog-to-digital converter," IEEE Trans. Circuits Syst. I, Reg. Papers, vol. 51, no. 1, pp. 130-139, Jan. 2004.

[8] J. Elbornsson, F. Gustafsson, and J.-E. Eklund, "Blind adaptive equalization of mismatch errors in a time-interleaved A/D converter system," IEEE Trans. Circuits Syst. I, Reg. Papers, vol. 51, no. 1, pp. 151-158, Jan. 2004

[9] J. Elbornsson, F. Gustafsson, and J.-E. Eklund, "Blind equalization of time errors in a time-interleaved ADC system," IEEE Trans. Signal Process., vol. 53, no. 4, pp. 1413-1424, Apr. 2005.

[10] S. Huang and B. Levy, "Adaptive blind calibration of timing offset and gain mismatch for two-channel time-interleaved ADCs," IEEE Trans. Circuits Syst. I, Reg. Papers, vol. 53, no. 6, pp. 1278-1288, Jun. 2006.

[11] S. Huang and B. Levy, "Blind calibration of timing offsets for fourchannel time-interleaved ADCs," IEEE Trans. Circuits Syst. I, Reg. Papers, vol. 54, no. 4, pp. 863-876, Apr. 2007.

[12] A. Haftbaradaran and K. Martin, "A background sample-time error calibration technique using random data for wide-band high-resolution time-interleaved ADCs," IEEE Trans. Circuits Syst. II, Exp. Briefs, vol. 55, no. 3, pp. 234-238, Mar. 2008.

[13] C.-Y. Wang and J.-T. Wu, "A background timing-skew calibration technique for time-interleaved analog-to-digital converters," IEEE Trans. Circuits Syst. II, Exp. Briefs, vol. 53, no. 4, pp. 299-303, Apr. 2006.

[14] Y.-C. Jenq, "Perfect reconstruction of digital spectrum from nonuniformly sampled signals," IEEE Trans. Instrum. Meas., vol. 46, no. 3, pp. 649-652, Jun. 1997

[15] Y. C. Eldar and A. V. Oppenheim, "Filterbank reconstruction of bandlimited signals from nonuniform and generalized samples," IEEE Trans. Signal Process., vol. 48, no. 10, pp. 2864-2875, Oct. 2000.

[16] H. Johansson and P. Lowenborg, "Reconstruction of nonuniformly sampled bandlimited signals by means of digital fractional delay filters," IEEE Trans. Signal Process., vol. 50, no. 11, pp. 2757-2767, Nov. 2002.

[17] R. S. Prendergast, B. C. Levy, and P. J. Hurst, "Reconstruction of bandlimited periodic nonuniformly sampled signals through multirate filter banks," IEEE Trans. Circuits Syst. I, Reg. Papers, vol. 51, no. 8, pp. 1612-1622, Aug. 2004.

[18] H. Johansson, P. Lowenborg, and K. Vengattaramane, "Least-squares and minimax design of polynomial impulse response FIR filters for reconstruction of two-periodic nonuniformly sampled signals," IEEE Trans. Circuits Syst. I, Reg. Papers, vol. 54, no. 4, pp. 877-888, Apr. 2007.

[19] S. Tertinek and C. Vogel, "Reconstruction of two-periodic nonuniformly sampled band-limited signals using a discrete-time differentiator and a time-varying multiplier," IEEE Trans. Circuits Syst. II, Exp. Briefs, vol. 54, no. 7, pp. 616-620, Jul. 2007.
[20] S. Tertinek and C. Vogel, "Reconstruction of nonuniformly sampled bandlimited signals using a differentiator-multiplier multiplier cascade," IEEE Trans. Circuits Syst. I, Reg. Papers, vol. 55, no. 8, pp. 2273-2286, Sep. 2008.

[21] T. Kailath, A. Sayed, and B. Hassibi, Linear Estimation, ser. Prentice Hall Information and System Sciences Series. Upper Saddle River, NJ: Prentice-Hall, 2000.

[22] B. D. O. Anderson and J. B. Moore, Optimal Filtering (Dover Books on Engineering), 1st ed. New York: Dover, 2005.

[23] E. Larsson, M. Mossberg, and T. Söoderström, "An overview of important practical aspects of continuous-time ARMA system identification," Circuits Syst. Signal Process., vol. 25, no. 1, pp. 17-46, 2006.

[24] K. Mahata and M. Fu, "On the indirect approaches for CARMA model identification," Automatica, vol. 43, no. 8, pp. 1457-1463, Aug. 2007.

[25] D. Pham, "Estimation of continuous-time autoregressive model from finely sampled data," IEEE Trans. Signal Process., vol. 48, no. 9, pp. 2576-2584, Sep. 2000.

[26] M. Mossberg, "Estimation of continuous-time stochastic signals from sample covariances," IEEE Trans. Signal Process., vol. 56, no. 2, pp. 821-825, Feb. 2008.

[27] K. Mahata and M. Fu, "Modelling continuous-time processes via input-to-state filters," Automatica, vol. 42, no. 7, pp. 1073-1084, Jul. 2006.

[28] K. Yao and J. Thomas, "On some stability and interpolatory properties of nonuniform sampling expansions," IEEE Trans. Circuits Theory, vol. CT-14, no. 4, pp. 404-408, Dec. 1967.

[29] P. Vaidyanathan, Multirate Systems and Filterbanks. Englewood Cliffs, NJ: Prentice-Hall, 1993.

[30] A. Ben-Israel and T. N. E. Greville, Generalized Inverses: Theory and Applications, ser. CMS Books in Mathematics/Ouvrages de Mathématiques de la SMC, 15, 2nd ed. New York: Springer-Verlag, 2003.

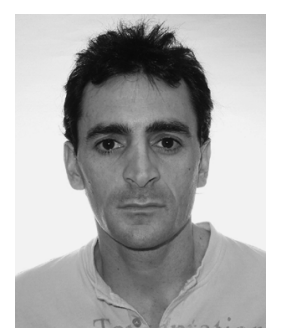

Damián Marelli received the B.Sc. degree in electronics engineering from the Universidad Nacional de Rosario, Rosario, Argentina, in 1995 and the Ph.D. degree in electrical engineering and the B.Sc.(Hons.) degree in mathematics from the University of Newcastle, Callaghan, Australia, in 2003.

In 2003, he was a Research Associate with the School of Electrical Engineering and Computer Science, University of Newcastle. In 2004 and 2005, he held a Postdoctoral Research Fellowship in the Laboratoire d'Analyse Topologie et Probabilités, CNRS/Université de Provence, Aix-en-Provnce, France. Since 2006, he has been a Research Academic with the ARC Centre for Complex Dynamic Systems and Control, University of Newcastle, where he is also with the School of Electrical Engineering and Computer Science. He held an Intra-European Marie Curie Fellowship in the Faculty of Mathematics, University of Vienna, Vienna, Austria, from 2007 to 2008. His main research interests include multirate signal processing, time-frequency analysis, system identification, and statistical signal processing.

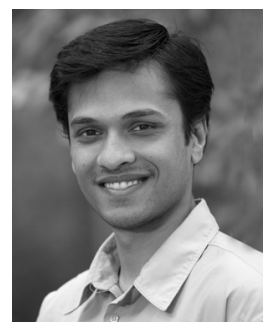

Kaushik Mahata received the Ph.D. degree in signal processing from Uppsala University, Uppsala, Sweden, in 2003 .

Since 2004, he has been with the School of Electrical Engineering and Computer Science, University of Newcastle, Callaghan, Australia. His research interests include statistical signal processing, system identification, and spectral analysis. 


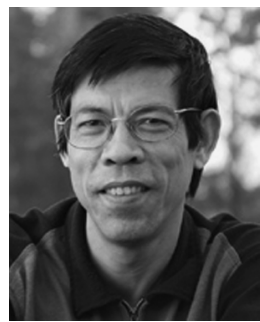

Minyue Fu (S'84-M'87-SM'94-F'04) received the B.S. degree in electrical engineering from the University of Science and Technology of China, Hefei, China, in 1982 and the M.S. and Ph.D. degrees in electrical engineering from the University of Wisconsin, Madison, in 1983 and 1987, respectively.

From 1983 to 1987, he held a teaching assistantship and a research assistantship at the University of Wisconsin. He was a Computer Engineering Consultant with Nicolet Instruments, Inc., Madison, WI, in 1987. From 1987 to 1989, he was an Assistant Professor with the Department of Electrical and Computer Engineering, Wayne State University, Detroit, MI. For the summer of 1989, he was with the
Universite Catholique de Louvain, Louvain-la-Neuve, Belgium. He joined the University of Newcastle, Callaghan, Australia, in 1989, where he is currently a Chair Professor in electrical engineering and the Head of the School of Electrical Engineering and Computer Science. He was a Visiting Associate Professor with the University of Iowa, Iowa City, in 1995-1996 and a Senior Fellow/Visiting Professor with Nanyang Technological University, Singapore, Singapore, in 2002. He holds a Changjiang Visiting Professorship at Shandong University, Jinan, China, and visiting positions at South China University of Technology, Guangzhou, China, and Zhejiang University, Hangzhou, China. His main research interests include control systems, signal processing, and communications.

Dr. Fu is currently an Associate Editor for the IEEE TRANSACTIONS ON AutOMATIC CONTROL, Automatica, and Optimization and Engineering. 